\title{
Automatic DWT2 thresholding based segmentation of the pigmented skin lesions in dermatoscopic images
}

\author{
Neda Razazzadeh ${ }^{1} *$, Mehdi Khalili ${ }^{2}$ \\ ${ }^{I}$ M.S. Student, Dept. Computer and Informatics Engineering, Payame Noor University, Gheshm, Iran \\ ${ }^{2}$ Assistant professor, Dept. Computer and Informatics Engineering, Payame Noor University, Tehran, Iran \\ *Corresponding author E-mail: N_Razazzadeh@yahoo.com
}

Copyright () 2014 Neda Razazzadeh and Mehdi Khalili. This is an open access article distributed under the Creative Commons Attribution License, which permits unrestricted use, distribution, and reproduction in any medium, provided the original work is properly cited.

\begin{abstract}
The segmentation is the most important step to automatic diagnosis of the skin lesions. In this paper, a DWT2 thresholding based segmentation of dermatoscopic images has been proposed to diagnose of the pigmented skin lesions. In the proposed method, first, the image is converted to YUV channels and after denoising and contrast enhancement of the second channel of the converted image, it is decomposed to wavelet transform in two levels. Then, to more specificity and accuracy of segmentation, the Otsu's thresholding method is applied on each sub-band of the second level of decomposed image and four thresholds are achieved. Subsequently, using adding all thresholds a new threshold is obtained and applied on the second level reconstructed image to achieve a binary image. Finally, post-processing is applied on this binary image using algorithms of morphological reconstructions, to increase the sensitivity. The experimental results show that the proposed method increases the accuracy to $90.97 \%$, and specificity to $99.76 \%$, compared with the other existing methods.
\end{abstract}

Keywords: DWT2, Morphological Reconstructions Algorithms, Otsu's Thresholding, Pigmented Skin Lesions, Segmentation

\section{Introduction}

Pigmented skin lesions include both, benign and malignant forms [1]. Malignant melanoma is the most dangerous human skin disease. It is the deadliest form of all skin cancers and arises from cancerous growth in pigmented skin lesion. If early recognized, the melanoma can be recovered completely. On the contrary, an invasive malignant melanoma reduces drastically the chance for the patient to survive because other effective therapeutic procedures are presently lacking [2]. Early diagnosis is of fundamental importance to improve the patient prognosis; nevertheless discriminating benign from malignant skin lesions has been proven to be a challenging task [1]. A recent advance in diagnosis of melanomas is the emergence of dermoscopy, also known as epiluminescence microscopy. Dermoscopy is a noninvasive diagnostic technique that consists in the examination of skin lesions with a dermoscope, which is a hand held optical device that typically consists of a magnifying lens and a light source, used to illuminate the skin. Usually, a transparent plate and liquid medium is placed between the instrument and the skin, allowing the inspection of a lesion, in the upper layers of the skin, in great detail [3]. Surface microscopy involves a layer of oil between the lesion and the optics. Air bubbles can be trapped in this layer [4]. Dermoscopy yields $10 \%-27 \%$ higher sensitivity than clinical diagnosis, significantly improving the accuracy of dermatologists when diagnosing melanoma. Yet, dermoscopic diagnosis remains subjective and is therefore associated with poor reproducibility. Because of this there has been a significant increase in interest in the development of automatic digital dermatoscopic image analysis methods over the last decade [5]. The standard approach in automatic dermoscopic image analysis has usually three stages: 1) image segmentation 2) feature selection and extraction, and 3) lesion classification [6].

For example, in order to achieve an effective way to identify skin cancer at an early stage without performing any unnecessary skin biopsies, in [7], feature extraction (as an essential-weapon to analyze an image appropriately) is considered using analyzing based unsupervised segmentation techniques of melanoma skin lesions images. In this work, feature extraction is based on the so-called ABCD-rule of dermatoscopy. 
Also, in [8], two hybrid techniques have been presented for the classification of the skin images to predict it if exists. The proposed hybrid techniques of this work consist of three stages, namely, feature extraction, dimensionality reduction, and classification;

On the other hand, due to different shapes and colors of dermoscopic images, accurate skin lesion segmentation is the most important stage of all [6].

In [9] a segmentation method is proposed in which the median filter of size 7 is used for image denoising. The resulted image from preprocessing stage, converted to grey scales. Then applied the Otsu method which is a technique based on pixel segmentation. An opening operation (i.e. erosion followed by a dilatation) and afterwards, a closing operation (i.e. dilatation followed by an erosion) with structured elements of size 3 are applied to manipulated image. Also algorithm of connected components labeling with 8- connectivity applied to binary images and biggest objects kept. Finally the image processed with a hole filling algorithm.

In [10], the authors have proposed a modification of wavelet approximation coefficients as the easiest way for edge detection. In this research two methods are evaluated: 1) Approximation coefficients replaced by zeros to remove low frequencies from the image (the image is reconstructed using only the remaining wavelet coefficients); 2) Modification of approximation coefficients by simple edge detectors such as Canny, Sobel, and Prewitt for applying to the obtained approximation coefficients in the first level of decomposition (the image is reconstructed from remaining and modified approximation coefficients).

Although in $[9,10]$ the segmentation properties such as specificity and accuracy are performed acceptable, but in a wide range of images they are not satisfactory. To overcome this problem, in this paper, an improved segmentation method is proposed in which first the image is converted to YUV channels form RGB color space. After denoising and contrast enhancement of $U$ channel, it is decomposed to two levels by wavelet transformation. Then, Otsu's thresholding is applied on the second level sub-bands of decomposed image and four thresholds are achieved, to more specificity and accuracy of segmentation. These thresholds are added together to obtain a new threshold T. After that, the second level of decomposed image is reconstructed and then converted to a binary image by applying T. The high sensitive segmented image is obtained after applying algorithms of morphological reconstruction on the binary image.

\section{Basic theories}

\subsection{Two dimensional discrete wavelet transform (DWT2)}

The discrete wavelet transform is a very useful tool for signal analysis and image processing, especially in multiresolution representation. The multi-resolution representation can provide a simple method for expounding the information about images. The two-dimensional discrete wavelet transform can decompose an image into 4 different resolutions of sub-bands [11]. Each one of these sub-bands are produced with the half size of the original image, corresponding to high frequencies in the horizontal direction and low frequencies in the vertical direction HL (horizontal details), low frequencies in the horizontal direction and high frequencies in the vertical direction LH (vertical details), high frequencies in both directions HH (diagonal details) and low frequencies in both directions LL (approximation coefficients) [12,13].

\subsection{Otsu's thresholding}

Otsu's thresholding is one of the oldest methods in the image segmentation. It is treated as a statistical method according to probabilistic implemented. It should be noted that the method Otsu is one of the best ways automatic threshold level. The basic principle in Otsu method is divided into two categories, such as foreground and the background. The threshold is obtained by finding automatic maximum contrast between the layers [14].

Otsu based on the pixel density contrast. Otsu method in the threshold level iterating through all possible limit values and calculate the behavior of the spreading the pixels levels around each side of the threshold, any pixel may fall either in the foreground or background [14].

\subsection{YUV color space}

Color images have more information than grey-level images which results in more reliable separation of foreground regions from background in color image segmentation algorithms. It has been recognized that selection of an appropriate color space produces more perceptually effective segmentation results. In particular, segmentation in YUV spaces produces more favorable results than the RGB space [15]. The YUV color space is used in European TV transmissions [16]. The chrominance components (U and V) are explicitly separated from the luminance (Y) component in the YUV model. Second, the YUV space is typically used in most image and video coding standards and therefore the computation burden for format transformation will be avoided in the edge extraction procedure. The latter property is very attractive for real-time image processing systems [17]. It is also able to reduce the inter-dependencies of the RGB components. The transform from RGB to YUV is as follow [16]: 
$\left[\begin{array}{c}\mathrm{Y} \\ \mathrm{U} \\ \mathrm{V}\end{array}\right]=\left[\begin{array}{ccc}0.299 & 0.587 & 0.114 \\ -0.147 & -0.289 & 0.436 \\ 0.615 & -0.515 & -0.100\end{array}\right]\left[\begin{array}{c}\mathrm{R} \\ \mathrm{G} \\ \mathrm{B}\end{array}\right]$

\section{The proposed segmentation framework}

Block diagram of the proposed method for automatic segmentation of pigmented skin lesions is shown in Fig.1. Three pre-processing, segmentation and post-processing of the proposed scheme are detailed as follows:

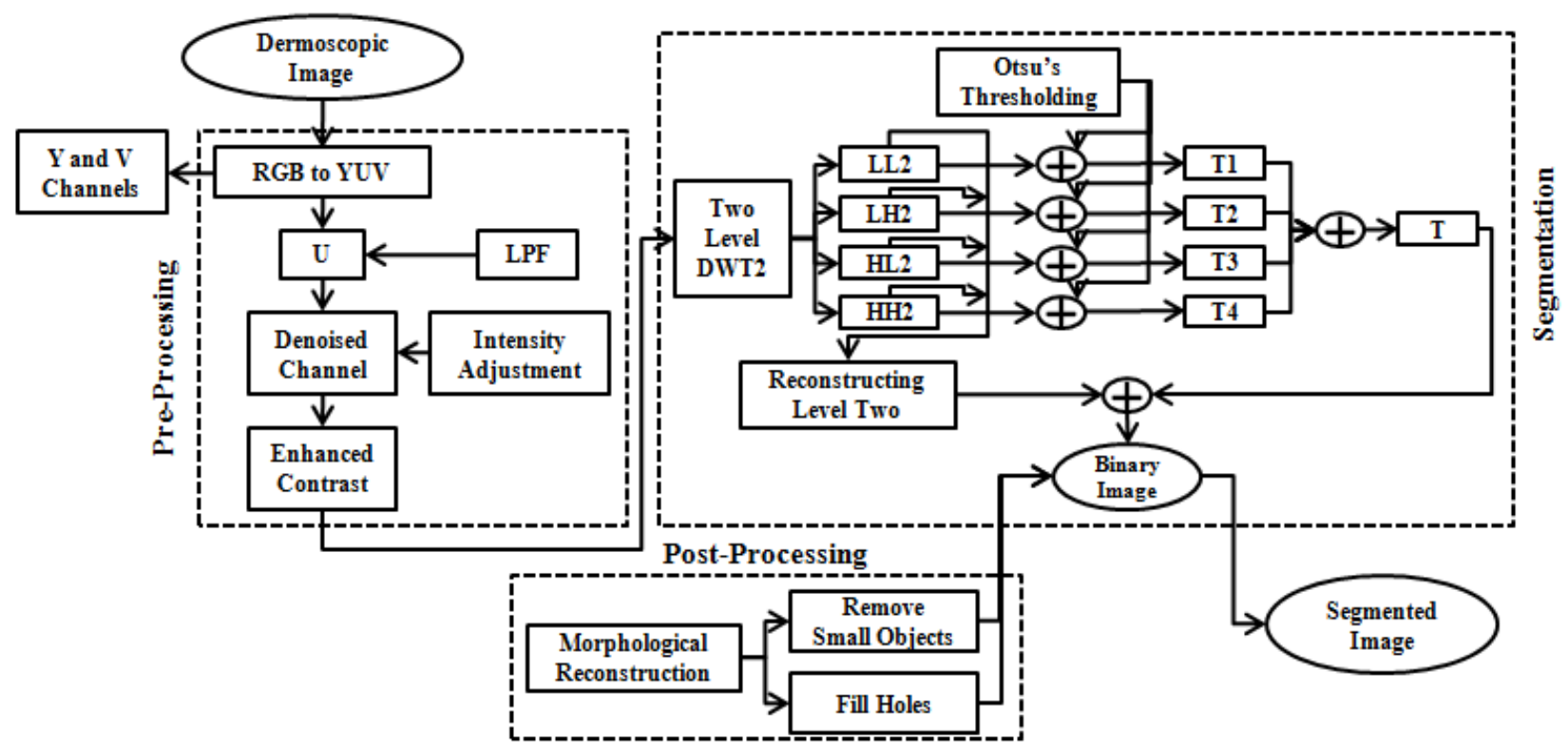

Fig. 1: Block Diagram of the Proposed Algorithm.

\section{Pre-Processing steps:}

1) Convert the dermoscopic image to YUV color space from RGB and select U channel as the target channel, because of its high clearness in showing the lesion.

2) Apply low past filtering (LPF) of furrier domain on U channel of converted dermatoscopic image to denoise the effects of hair, skin lines, blood vessels, and air bubbles.

3) Enhance the contrast of denoised image using intensity adjustment.

\section{Segmentation steps:}

In this step, wavelet decomposition and Otsu's thresholding are used for classifying image pixels to lesion (white area) and skin (background area), to produce a binary image. The segmentation steps are described as follows:

4) Decompose the denoised U channel in two levels wavelet transform.

5) Apply Otsu' thresholding method on each second level sub-band to obtain four threshold values: T1, T2, T3 and T4.

6) Add all of the thresholds to achieve new threshold $\mathrm{T}$.

7) Reconstruct the second level decomposed sub-bands.

8) Apply the new threshold $\mathrm{T}$ on the reconstructed sub-bands to obtain a binary image as follows:

$p(i, j)= \begin{cases}1 & r(i, j)<T \\ 0 & \text { Otherwise }\end{cases}$

Where $r(i, j)$ and $p(i, j)$ respectively are the images pixel values of reconstructed from second level sub-bands and binary.

\section{Post-Processing steps:}

This step uses the related morphological reconstruction algorithms to increase the sensitivity of proposed method as follows:

9) Determine the connected components and compute the area of each component to remove the small objects.

10) Fill holes in the binary image 


\section{Analysis of the proposed algorithm and experimental results}

The proposed scheme has been conducted on 319 dermatoscopic images which belong to standard data base from two university hospitals (University of Naples, Italy, and University of Graz, Austria) and stored in BMP format. All cases were diagnosed on the basis of histopathological examination of biopsy materia. The images include 188 Clark nevi, 56 Reed nevi and 75 melanomas, (including 23melanoma in situ) [18].

All of the experiments were implemented using MATLAB 7.12. Also, biorthogonal spline (B-spline) wavelet filters was used for computation of the wavelet transforms. Cause of using B-spline function wavelet is that, B-spline functions, do not have compact support, but are orthogonal and have better smoothness properties than other wavelet functions [19]. It has to be mentioned that, in post-processing step, the areas smaller than 800 pixels and were diagnosed as the lesion wrongly are removed and also the holes that were diagnosed as the background wrongly are filled.

To provide objective judgment of the segmentation properties, sensitivity, specificity and accuracy, are applied to the proposed method.

A sensitivity measure is the proportion of actual lesion pixels that are correctly identified as such; specificity measures means the proportion of background skin pixels that are correctly identified; and accuracy determines the true value, the repeatability or reproducibility of the measurement, the proximity of measurement to the precision results $[1,20]$. They are defined as follows [21]:

$$
\begin{aligned}
& \text { Sensitivity }=\frac{T P}{T P+F N} \\
& \text { Specificity }=\frac{T N}{T N+F P} \\
& \text { Accuracy }=\frac{T P+T N}{T P+F N+T N+F P}
\end{aligned}
$$

where TP (true positive) is lesion pixels correctly classified as lesion; FP (false positive) is skin pixels incorrectly identified as lesion; and TN (true negative) is skin pixels correctly identified as skin; FN (false negative) is lesion pixels incorrectly identified as Skin [20].

Table 1 shows the obtained results of 10 implemented sample images. As it can be seen, the minimum percentage of sensitivity is 76.78 , all of the specificity values are higher than $99.00 \%$ and minimum percentage of accuracy is equal to 91.07 .

Table 1: The Obtained Results of 10 Implemented Sample Images

\begin{tabular}{llllllll}
\hline Image & TP & TN & FP & FN & Sensitivity (\%) & Specificity (\%) & Accuracy $(\%)$ \\
\hline 1 & 110025 & 156055 & 141 & 16161 & 87.19 & 99.90 & 94.22 \\
2 & 79255 & 135453 & 53 & 13384 & 85.55 & 99.96 & 94.11 \\
3 & 174873 & 271603 & 2880 & 40882 & 81.05 & 98.95 & 91.07 \\
4 & 98863 & 147514 & 4 & 13219 & 88.20 & 99.99 & 94.90 \\
5 & 41313 & 80287 & 4 & 7624 & 84.42 & 99.99 & 94.09 \\
6 & 51259 & 102497 & 855 & 6693 & 88.45 & 99.17 & 95.32 \\
7 & 153413 & 179272 & 1803 & 11352 & 93.11 & 99.00 & 96.19 \\
8 & 6468 & 18797 & 0 & 1956 & 76.78 & 100.0 & 92.81 \\
9 & 102231 & 191660 & 1137 & 13972 & 87.97 & 99.41 & 95.11 \\
10 & 106282 & 139000 & 0 & 18520 & 85.16 & 100.0 & 92.97 \\
\hline
\end{tabular}

Also, to illustrate the quality of the proposed method, we have simulated it on all of the 319 dermatoscopic images. The obtained results show that average values for sensitivity is $76.81 \%$, for specificity is $99.76 \%$ and for accuracy is equal to $90.97 \%$. These results reveal that the proposed segmentation method can separate pigmented lesion from surrounding skin very well.

After achieving desired results in satisfying segmentation properties, to show the improvement of the proposed scheme, the proposed earlier algorithms [9, 10], under the same conditions were implemented on the same 319 dermatoscopic images. The related performance comparisons are listed in table 2. As it is obvious, our proposed method yields to higher results in sensitivity, specificity and accuracy.

Table 2: The Comparison Results of the Proposed Scheme with the Earlier Works

\begin{tabular}{llll}
\hline Segmentation Algorithms & Sensitivity (\%) & Specificity (\%) & Accuracy (\%) \\
\hline$[9]$ & 78.72 & 94.26 & 87.14 \\
{$[10]$} & 42.92 & 90.20 & 70.79 \\
proposed & 76.81 & 99.76 & 90.97 \\
\hline
\end{tabular}


Fig. 2 indicates four samples of implemented dermatoscopic images, to better view the performance of our proposed method. Fig. 2 (b) shows the hand-drawn areas of Fig. 2 (a), which are determined by five expert dermatologists [18]. These extracted borders are traced in Fig. 2 (c) and compared with the algorithms [9, 10] and our proposed scheme in Figs. 2 (d-f). Clearly, it is visible the detection error in $[9,10]$ is high and borders detection are difficult; while our proposed scheme can recognize the lesion borders automatic as well.

(a)
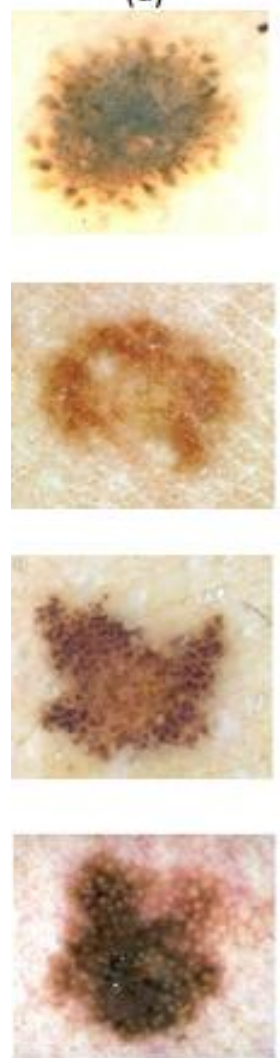

(b)
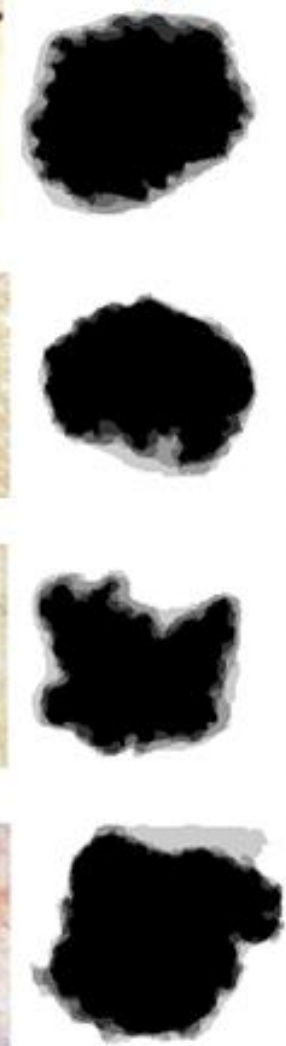

(c)
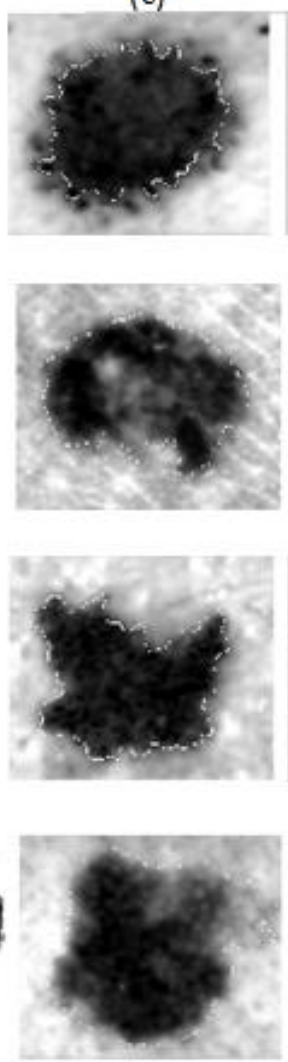

(d)
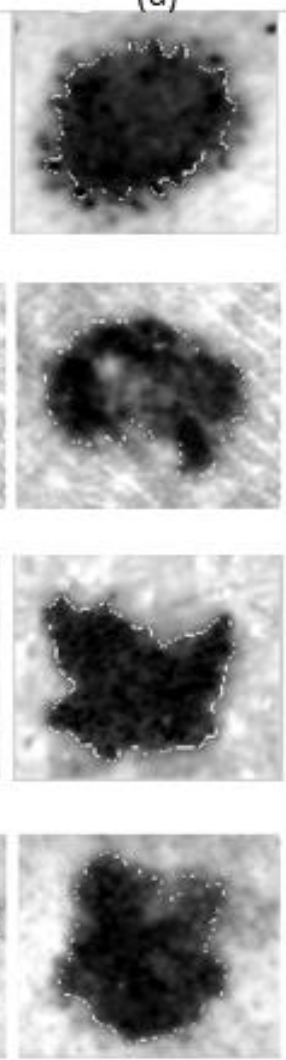

(e)
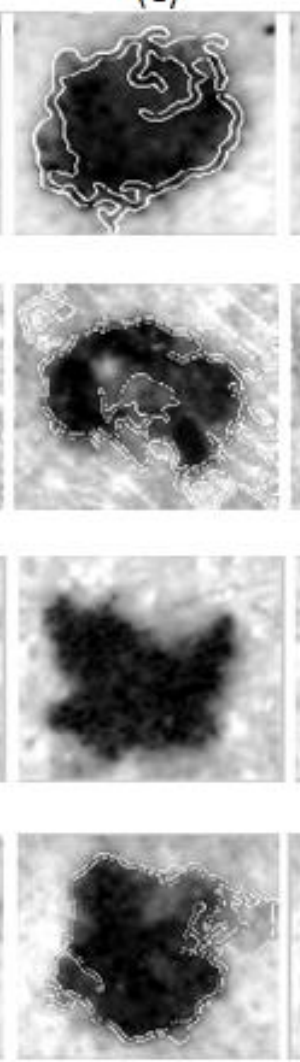

(f)
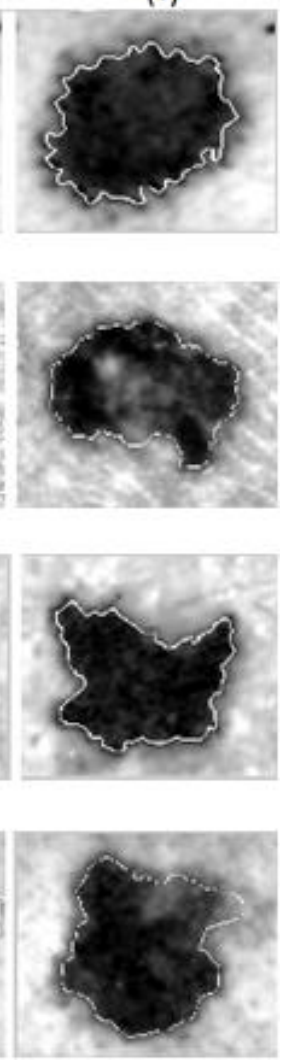

Fig. 1: Four Samples of Implemented Dermatoscopic Images; (A) Original Images; (B) Hand-Drawn Areas of Original Images Which are Determined by Five Expert Dermatologists; (C) Traced Extracted Borders of Hand-Drawn Areas; (D) the Extracted Borders From [9]; (E) the Extracted Borders From [10]; and (F) the Extracted Borders from the Proposed Method.

\section{Conclusion}

In this paper, an improved automatic DWT2 thresholding-based segmentation of the pigmented skin lesions in dermatoscopic images is proposed in which $U$ channel of the converted dermatoscopic image, after denoising and contrast enhancement is decomposed by wavelet transform in two levels. Subsequently, four thresholds are obtained using applying the Otsu's thresholding method on each sub-band of the second level, to more specificity and accuracy. Adding these four thresholds yields a new threshold to applying on the second level reconstructed image and finally achieving a binary image. Moreover, to increase the sensitivity, post-processing is applied on the binary image (using algorithms of morphological reconstructions). Experimental results have shown that the proposed scheme not only satisfies segmentation properties as well and increases the accuracy to $90.97 \%$, and specificity to $99.76 \%$, rather has improved the obtained results of implemented algorithms [9, 10] in the operation of common segmentation characteristics, clearly.

\section{References}

[1] H. Sood, M. Shukla, "Various Techniques for Detecting Skin Lesion: A Review", International Journal of Computer Science and Mobile Computing, Vol.3, Issue.5, pp. 905-912, May- 2014.

[2] S. P. B., S. Pande, "Segmentation of Melanoma Skin Cancer Images Based on Clustering Techniques", Research Journal of Computer Systems Engineering-RJCSE, Vol.02, Issue. 05, October-December 2011.

[3] M. Zortea, S. O. Skrovseth, T. R. Schopf, H. M. Kirchesch, and F. Godtliebsen, "Automatic Segmentation of Dermoscopic Images by Iterative Classification", International Journal of Biomedical Imaging, Vol. 2011, pp.1-19, 2011. http://dx.doi.org/10.1155/2011/972648.

[4] H. Talbot and L. Bischof, "An Overview of the Polartechnics SolarScan Melanoma Diagnosis Algorithms", Proceedings of the APRS Workshop on Digital Image Computing, 2003, pp.33-38.

[5] J. J. P. Rajam, A. A. H. Thasneem, "Detection of Skin Lesions in Dermoscopic Images", International Journal of Recent Development in Engineering and Technology, Vol.2, Special Issue 3, pp. 193-198, February 2014. 
[6] F. Xie, A. C. Bovik, "Automatic Segmentation of Dermoscopy Images Using Self-generating Neural Networks Seeded by Genetic Algorithm”, Pattern Recognition, vol.46, pp.1012-1019, 2013. http://dx.doi.org/10.1016/j.patcog.2012.08.012.

[7] Md. A. H. Bhuiyan, I. Azad, Md. K. Uddin, "Image Processing for Skin Cancer Features Extraction", International Journal of Scientific \& Engineering Research, Vol.4, Issue 2, February-2013.

[8] M. Elgamal, "Automatic Skin Cancer Images Classification", (IJACSA) International Journal of Advanced Computer Science and Applications, Vol.4, No. 3, 2013. http://dx.doi.org/10.14569/IJACSA.2013.040342.

[9] D. Ruiz, V. Berenguer, A. Soriano, B. Sánchez, "A Decision Support System for the Diagnosis of Melanoma: A Comparative Approach", Expert Systems with Applications, Vol. 38, pp.15217-15223, 2011. http://dx.doi.org/10.1016/j.eswa.2011.05.079.

[10] J. Petrova, E. Hostalkova, "Edge Detection in Medical Image Using the Wavelet Transform", Report of research, Department of Computing and Control Engineering, Czech Public, 2011.

[11] G. Nagendhar, D. Rajani, C. V. Sonagiri , V. Sridhar, "Text Localization in Video Data Using Discrete Wavelet Transform", International Journal of Innovative Research in Science, Engineering and Technology, Vol. 1, Issue 2, December 2012.

[12] C. R. Jung "Combining Wavelets and Watersheds for Robust Multiscale Image Segmentation", Image and Vision Computing, Vol.25, pp. 2433, 2007. http://dx.doi.org/10.1016/j.imavis.2006.01.002.

[13] M. Khalili, D. Asatryan, "Colour Spaces Effects on Improved Discrete Wavelet Transform-based Digital Image Watermarking Using Arnold Transform Map”, IET Signal Process, Vol. 7, No. 3, pp. 1-11, 2013. http://dx.doi.org/10.1049/iet-spr.2012.0380.

[14] N. Kh. E. Abbadi and A. H. Miry, "Automatic Segmentation of Skin Lesions Using Histogram Thresholding”, Journal of Computer Science 10, Vol.4, pp.632-639, 2014.

[15] F. A. Tab, G. Naghdy and A. Mertins, "Scalable Multiresolution Color Image Segmentation", Visual Communications and Image Processing, Vol.5960, 2005.

[16] N. M. Kwok, Q. P. Ha, G. Fang, "Effect of Color Space on Color Image Segmentation”, Image and Signal Processing. CISP '09. 2nd International Congress on, Tianjin, pp. 1-5, 2009.

[17] J. Fan, D. K. Y. Yau, A. K. Elmagarmid, and W. G. Aref, "Automatic Image Segmentation by Integrating Color-Edge Extraction and Seeded Region Growing", IEEE Trans. Image Processing, Vol. 10, NO. 10, october 2001.

[18] H. Iyatomi, H. Oka, M. Saito, A. Miyake, M. Kimoto, J. Yamagami, S. Kobayashi, A. Tanikawa, M. Hagiwara, K. Ogawa, G. Argenziano, H. P. Soyer and M. Tanaka, "Quantitative Assessment of Tumour Extraction from Dermoscopy Images and Evaluation of Computer-based Extraction Methods for an Automatic Melanoma Diagnostic System”, Melanoma Research, Vol.16, pp. 183-190, 2006. http://dx.doi.org/10.1097/01.cmr.0000215041.76553.58.

[19] L. Fan, T. Gao, "A Novel Blind Robust Watermarking Scheme Based on Statistic Characteristic of Wavelet Domain Coefficients", Proceedings of the International Conference on Signal Processing Systems, Tianjin, China, May 2009, pp. 121-125.

[20] H. Castillejos, V. Ponomaryov, L. N.-de-Rivera and V. Golikov, "Wavelet Transform Fuzzy Algorithms for Dermoscopic Image Segmentation", Computational and Mathematical Methods in Medicine, Vol. 2012, pp. 41-52, 2012. http://dx.doi.org/10.1155/2012/578721.

[21] J. Glaister, A. Wong, and D. A. Clausi, "Segmentation of Skin Lesions from Digital Images Using Joint Statistical Texture Distinctiveness", IEEE Trans. Biomedical Engineering. Vol. 61, Issue. 4, pp.1220 - 1230, 2014. http://dx.doi.org/10.1109/TBME.2013.2297622. 